\title{
Femtosecond lasers for countermeasure applications
}

\author{
G.C. Franssen*a, H.M.A. Schleijpen ${ }^{a}$, J.C. van den Heuvel ${ }^{a}$, H. Buersing ${ }^{b}$, B. Eberle ${ }^{b}$, \\ and D. Walter ${ }^{\mathrm{b}}$ \\ ${ }^{a}$ TNO Defence, Security and Safety, Oude Waalsdorperweg 63, The Hague, The Netherlands \\ ${ }^{\mathrm{b}}$ FGAN Reseach Institute for Optronics and Pattern Recognition, D-76275 Ettlingen, Germany
}

\begin{abstract}
In recent years, much advance in the field of high-power femtosecond laser technology has been made. The high pulse power of femtosecond laser systems leads to various interesting phenomena, such as a very high power density and the formation of a plasma in the propagation medium, which is usually air. The possible application of femtosecond lasers for infrared countermeasure (CM) applications, other than direct illumination of the detector, was suggested by several authors. The goal of these countermeasures is to divert missiles from their interception course. We quantitatively examined several suggested $\mathrm{CM}$ applications of femtosecond lasers from the literature. Our analyses show that application of femtosecond laser beams for dazzling of missile seeker heads is not in any way promising, neither via white-light generation in the ultra-short laser pulses nor via glowing plasma. In both these cases the generated light intensity is too low to successfully compete with the signal of a typical target. Taking into account literature reports, we conclude that the most promising application of femtosecond laser beams in the field of countermeasure applications seems to be related to inducing damage of the optical components of the seeker system.
\end{abstract}

Keywords: femtosecond lasers, optical countermeasures

\section{INTRODUCTION}

Recent advancements in the technology of femtosecond lasers have triggered many ideas for applications of this type of lasers as optical or infrared countermeasures. Femtosecond laser beams have a number of distinct properties when compared to pulsed lasers with pulse durations of the order of nano- or picoseconds. Some of these specific features, such as very high peak power, non-linear optical effects, and plasma filaments, may be useful for application in new countermeasure techniques. In this paper, we examine possible countermeasure applications of femtosecond lasers.

The paper is organized as follows. As an introduction to the topic, in section 2, we will discuss some general characteristics of femtosecond laser technology. After this, in section 3, potential applications of several aspects of femtosecond laser beams will be discussed in more detail. It is our purpose to describe potential applications and to give an impression of the feasibility of the considered techniques. This involves an analysis of relevant literature in order to identify the technical challenges which need to be solved. Important results are the uncertainties related to the potential countermeasure application. The aspects of femtosecond laser technology which will be discussed in the context of countermeasures are (i) the white-light spectral continuum, (ii) plasma emission, (iii) beam filamentation, (iv) highpower damaging of components, and (v) jamming of detectors.

\section{FEMTOSECOND LASER TECHNOLOGY}

In recent years, high-pulse-power femtosecond laser systems have become commercially available. Available systems include both container-size femtosecond lasers for military and/or large-scale scientific applications, and more modest table-top systems for laboratory applications. The former type is of most relevance in the context of potential countermeasures. It is worthwhile to mention here the container-size mobile femtosecond laser constructed in the framework of the Teramobile project, initiated by Centre National de la Recherche Scientifique (CNRS) and Deutsche Forschungsgemeinschaft (DFG) [1] (mainly used in scientific research) and the Terawatt\&THz (T\&T) mobile laboratory

Technologies for Optical Countermeasures VI, edited by David H. Titterton, Mark A. Richardson, Proc. of SPIE Vol. 7483, 748309 - @ 2009 SPIE · CCC code: 0277-786X/09/\$18 · doi: 10.1117/12.830575 
of Defense Research \& Development Canada (DRDC) [2] (intended explicitly for exploring defense applications of high-power femtosecond lasers). Typical parameters of the mobile femtosecond laser systems currently available are the following: $\Delta \mathrm{t}_{\text {pulse }} \approx 50-200 \mathrm{fs} ; \mathrm{E}_{\text {pulse }} \approx 1 \mathrm{~mJ}-1 \mathrm{~J} ;$ repetition rate $\approx 10 \mathrm{~Hz}-1 \mathrm{kHz} ; \lambda_{\text {central }} \approx 800 \mathrm{~nm}(\mathrm{Ti}: \mathrm{Sa}) ; \mathrm{P}_{\text {average }} \approx 10 \mathrm{~W}$; $\mathrm{P}_{\text {peak }} \approx 1$ TW. Please note that, due to the low repetition rate, the average power of a typical femtosecond is not very high. It is therefore preferable to refer to these systems as 'high-pulse-power' lasers rather than 'high-power' lasers. Another point of attention in the context of countermeasures is the rather low repetition rate of especially the femtosecond lasers with higher pulse powers. The $10 \mathrm{~Hz}$ repetition rate may not suffice to be effective in countermeasure

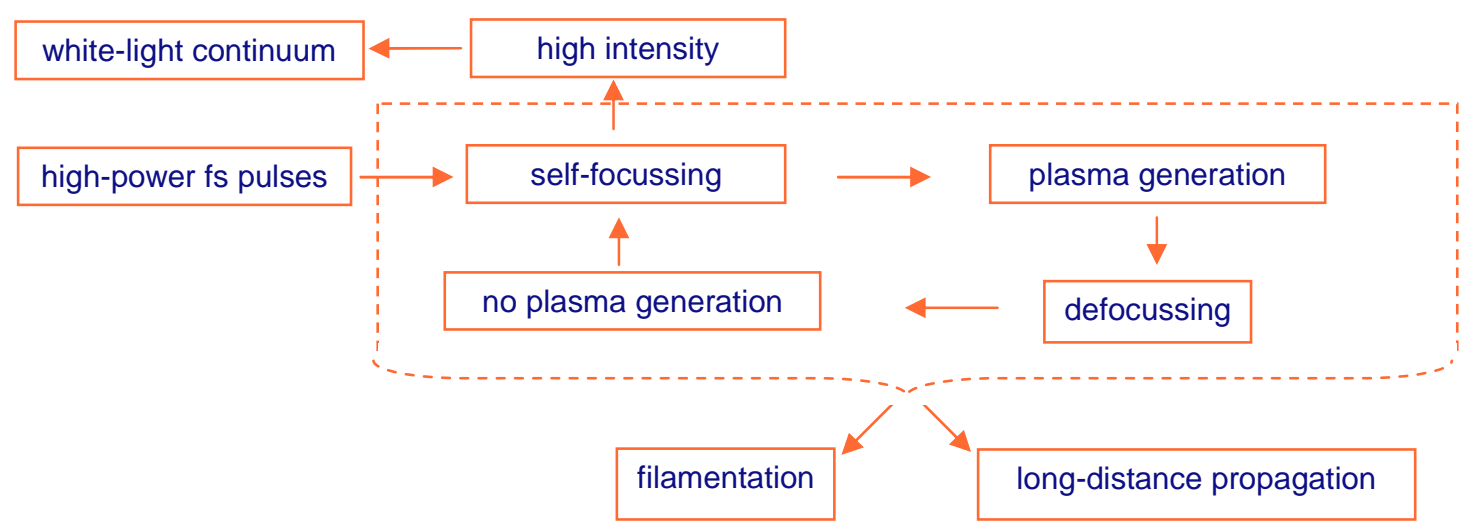

Fig. 1. Summary of physical effects relevant in femtosecond laser beams, and their mutual relations.

applications in which often pulse repetition rates of a few hundred $\mathrm{Hz}$ are required.

The high peak power of the femtosecond pulses, in the TW range or even higher, has important consequences for the beam properties. For convenience, the relevant effects are summarised in Fig. 1. First of all, due to the nonlinear behavior of the refractive index, the high light intensities lead to a significant variation of the refractive index across the beam profile in air. The nonlinearity of the index of refraction $n$ is expressed as [3]:

$$
n=n_{0}+n_{2} \cdot I \text {, }
$$

in which $n_{0}$ is the low-intensity index of refraction, $n_{2}$ is the nonlinear index of refraction, and $I$ is the light intensity.

The higher refractive index in the centre with respect to the outer region of the beam gives rise to a positive lensing effect (also known as 'Kerr lens') which focusses the light on the beam axis. This self-focussing process cannot be expected to go on indefinitely, and indeed it does not. Self-focussing leads to a build-up of light intensity at the beam axis up to a light intensity of about $4 \cdot 10^{13} \mathrm{~W} / \mathrm{cm}^{2}$ at which air becomes sufficiently (photo-)ionised to induce defocussing of the beam [4]. The defocussing is related to the fact that the higher electron density of ionised air brings about a lower refractive index and, in reverse analogy to the self-focussing effect discussed before, the laser beam will defocus. This, in turn, will lead to a lower light intensity and again increased self-focussing. The cyclic character of this process is indicated in Fig. 1. At sufficiently high light intensities, channels of light (so-called 'filaments') are formed. It should be added that the generated plasma is a potential source of radiation, either through recombination processes or via Planck radiation. Plasma radiation can also be generated via nonlinear interaction between light and plasma oscillations.

Several other physical effects in high-power femtosecond pulses which are potentially relevant for countermeasures are mentioned now. Long-distance propagation is the result of the influence of focussing and defocussing processes on the beam propagation. The cyclic focus-defocus process makes the beam differ essentially from the classic diverging Gaussian laser beam. In contrast, the femtosecond laser beam can propagate without divergence. Propagation distances up to hundreds of meters have been reported [5]. 
Filamentation of femtosecond laser beams, i.e. the partition of the beam into a number of narrow channels with diameters of the order of $100 \mu \mathrm{m}$, is directly related to the limited light intensity of a laser beam that can be supported by air (due to photo-ionisation and defocussing). When this threshold intensity is surpassed, the laser beam cross-section expands into a configuration with many narrow channels (the abovementioned filaments), which limits the intensity [6]. A large number of filaments, approximately equal to $I_{\text {laser }} / I_{\text {crit }}$ (in which $I_{\text {laser }}$ is the laser light intensity and $I_{\text {crit }}$ is the critical light intensity for self-focussing) can therefore be generated by a single laser source. The exact mechanisms of formation and evolution of the filaments are not yet very well understood. Filaments can travel long distances without divergence, as discussed in the preceding paragraph. However, control of the filaments in terms of position and temporal stability is only obtainable to some degree at present due to the high nonlinearity of the process.

The spectral characteristics of femtosecond pulses are considerably influenced by temporal variation of the intensity. In particular, the high intensity of the light in the centre of the pulse with respect to its onset and its trailing part has serious consequences. Indeed, the time-dependent refractive index associated with a passing pulse [as described by Eq. (1)] implies a time-dependent frequency shift [7]. In particular, the leading edge of a pulse is red shifted, while the trailing edge undergoes a blue shift. This process is referred to as self-phase modulation (SPM). SPM leads to spectral broadening. Also, in the case of a regular dispersion (with a higher phase velocity for larger wavelengths), temporal broadening is observed. The broadened spectrum covers the visible part of the spectrum and is therefore referred to as the white-light continuum (WLC). The continuum spectrum also extends into the infrared, where relatively low power levels are achieved.

\section{APPLICATION OF FEMTOSECOND LASERS IN COUNTERMEASURE TECHNOLOGY}

\subsection{White-light continuum}

The scenario we consider for the discussion of countermeasure applications of femtosecond lasers looks as follows: a missile has locked on to a plane and follows it at a certain distance, while a ground-based femtosecond laser system sends out a laser beam with the intention of jamming the tracking mechanism. The situation is illustrated in Fig. 2. In order to achieve jamming, the jamming signal should be considerably larger than the signature of the aircraft. A factor of 100 is a safe assumption. The signature of the plane can be assumed to be in the order of 10-1000 W/sr in the relevant spectral region, where the former number is related to emission from the front and the latter to emission from the rear of the plane.

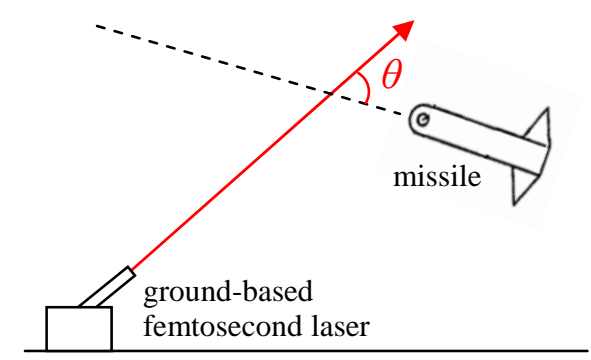

Fig. 2. Femtosecond laser beam directed at a seeker head under an angle $\theta$. The dashed line represents the missile line-of-sight towards the aircraft target, the red arrow is the femtosecond laser beam. A small angle $\theta$ allows for illuminating the seeker head with photons from the white-light continuum.

As was mentioned in section 2, the WLC of femtosecond laser pulses is related to the dependence of the refractive index on light intensity [as expressed by Eq.(1)], which is also responsible for self-focussing. The spectral characteristics of the WLC were determined by Kasparian et al. [8] for a typical femtosecond pulse with a peak power of $2 \mathrm{TW}$, and was 
observed to extend from $300 \mathrm{~nm}$ to $4.5 \mu \mathrm{m}$. Although the spectrum is very broad, it can be observed in Fig. 1 of [8] that most of the emitted power is still concentrated in the spectral region around the central wavelength of $800 \mathrm{~nm}$. For countermeasure applications we are interested in irradiating the seekerhead of a missile with radiation in a wavelength region in which they are sensitive in order to achieve jamming of the tracking mechanism. Assuming a region of 3-5 $\mu \mathrm{m}$ for the effective jamming wavelength, we see after integration of the relevant region that only $10^{-4}$ of the power contained by the WLC is potentially suitable for countermeasure purposes in the mid-wave IR.

Another limiting factor of the use of the WLC for countermeasure purposes is the high directionality of the laser light. In other words, WLC emission is not isotropic. As is shown in [9], the light travels mainly forward and in addition there is substantial backscattering. It is not possible to quantify these processes on the basis of the data from [9], but it is clear that scattering to the sides is negligible compared to forwardscattering and backscattering. Therefore, in order for WLC light to reach the seekerhead, the laser beam must be pointed quite precisely at the missile, nearly parallel to the missile line-of-sight. Based on [9], the angle $\theta$ in Fig. 2 should at least be below about $10^{\circ}$. Light from the laser beam passing in front of the missile at a distance ahead (not pointed into the seeker) will not easily be effective.

Based on the above, we can estimate the power incident on a seekerhead for a configuration in which the laser beam is directed towards the missile under an angle $\theta$, as illustrated in Fig. 2. Seeing that most of the light from the WLC is scattered along the direction of propagation [9], we use the approximation that all available photons propagate within the angle of divergence of the laser beam. In fact, part of the light undergoes backscattering. However, since this process, if accounted for, would only lead to a decrease of the estimated power on the seekerhead, we neglect it for our present estimations. Assuming $100 \mathrm{~mJ}$ pulses at a repetition rate of $100 \mathrm{~Hz}$, a total average power of $10 \mathrm{~W}$ is emitted by the laser. If we further assume an angle of $5 \mathrm{mrad}$ for the laser beam divergence (after Ref. [10], p.109), we obtain for a femtosecond laser located $100 \mathrm{~m}$ away from the missile an irradiance of $10 \mathrm{~W} \times 10^{-4} / 0.25 \mathrm{~m}^{2}=4 \cdot 10^{-3} \mathrm{~W} / \mathrm{m}^{2}$. As a second example, for a laser located $1000 \mathrm{~m}$ away from the missile we obtain an irradiance of $4 \cdot 10^{-5} \mathrm{~W} / \mathrm{m}^{2}$. These numbers should be compared with the aircraft signature. Assuming the aforementioned plane signature of 10-1000 W/sr and a distance from plane to missile of $1000 \mathrm{~m}$, we see that the plane signature at the position of the missile is $10^{-3}-10^{-5} \mathrm{~W} / \mathrm{m}^{2}$.

Since we need the jamming signal to be several orders of magnitude higher than the signal from the plane, we can conclude that the MWIR optical power provided by the WLC of the femtosecond laser pulse is not sufficient for effective countermeasures. In addition, it should be stressed that WLC emission is only available along the direction of beam propagation, which renders aiming issues crucial. Indeed, as the beam divergence is rather small, aiming precision should be high. Another potential problem is the narrow field-of-view of typical seekerheads, which makes effective irradiation of airborne missiles with a ground-based lasersystem practically impossible. Finally, the assumed pulse repetition rate of $100 \mathrm{~Hz}$ is ideally higher (in the order of $1 \mathrm{kHz}$ ), which would lower the pulse energy. This list of problems shows that the WLC of femtosecond laser pulses is not expected to be useful in countermeasure applications.

It is interesting to add here that white-light generation can also be achieved inside the optical components of, for example, the dome of a seeker system. The non-linear refractive index $n_{2}$ [see Eq. (1)] is approximately proportional to the density of the material. Since solids are more dense than air (by a factor of $\sim 10^{3}$ ), it is expected that the critical power density is much lower in optical materials than in air. This is confirmed in [11], in which white light generation is achieved in water and glass at much lower power than in air. It is claimed that $0.6 \mathrm{uJ}$ with $130 \mathrm{fs}$ pulsewidth is enough to create a filament in water. However, the light needs to propagate a certain distance in the material and it is at present unclear whether the propagation distance in for example the material of a missile dome with a thickness of a few millimetres is suffient to achieve white light generation from a normal-size beam. White-light generation in optical materials could present a way to evade blocking of the light by laser notch filters. However, it should be kept in mind that light generation may at least partly be due to material-specific fluorescence mechanisms. Indeed, at sufficiently high illumination intensities also photons at a relatively long wavelength of about $800 \mathrm{~nm}$ can excite photons in many materials via multiphoton absorption.

\subsection{Plasma emission of femtosecond laser beams}

We observed in section 2 that the generation of plasma in a high-power femtosecond laser beam is essential for some of its other characteristics, such as long-distance propagation and the related filamentation process. However, one can also 
imagine that the generated plasma may play a role in countermeasure applications. Glowing plasma can possibly act as a flare and divert the incoming missile from its original target by emitting suitable IR radiation, as indicated in Fig. 3. This principle was proposed in a patent from Bohn and Eckel [12]. However, the patent does not contain any quantitative analysis. We will provide such an analysis in the following.

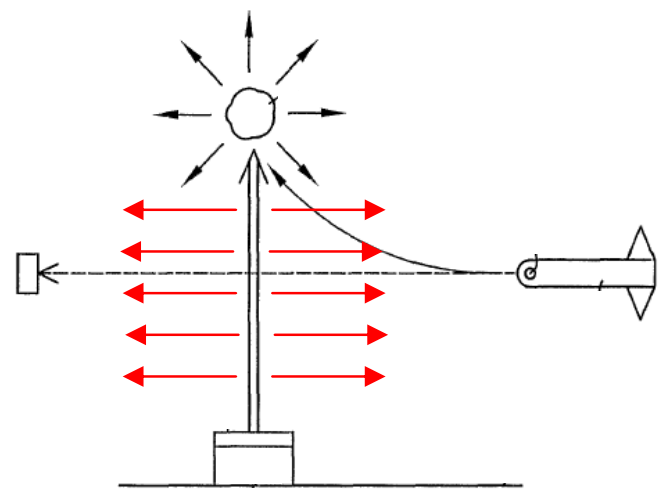

Fig. 3. Schematic representation of a scheme using a femtosecond laser beam to divert a missile by generating glowing plasma in the missile field-of-view. Red arrows indicate photon emission due to glowing plasma. The illustration is based on [12].

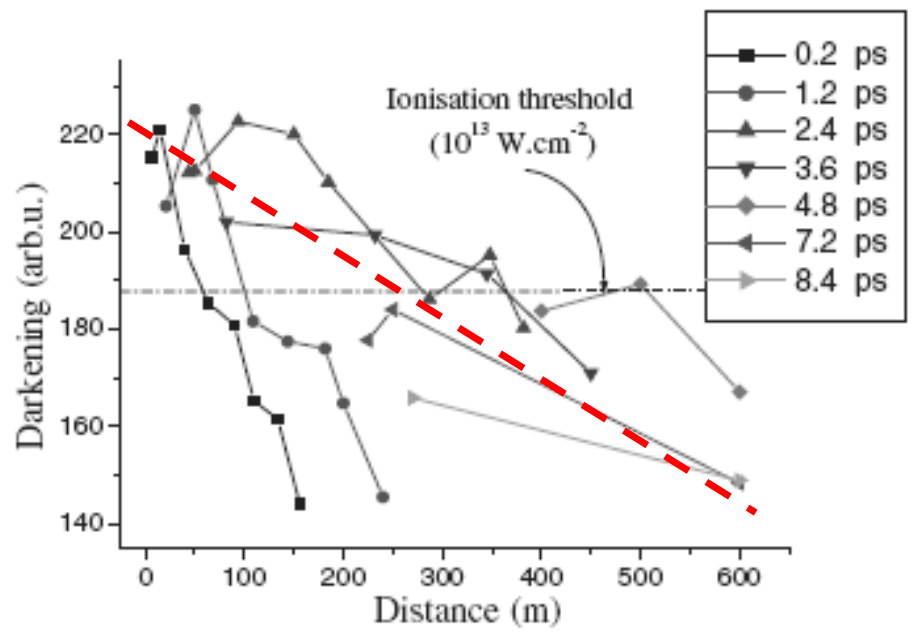

Fig. 4. Maximum light intensity (expressed as darkening of photographic paper) of a femtosecond laser as a function of distance for various pulse durations. The red dashed line indicates an intensity loss of $5 \%$ per $100 \mathrm{~m}$ [5]. Data are reprinted from [5] with permission from Elsevier.

The scenario we consider is similar to the scenario described in section 3.1, with a missile locked on to a plane which emits a 10-1000 W/sr signature. The plasma which is created in the path of the laser beam should be assumed to emit photons isotropically in the radial direction, contrary to Ref. [12] in which the authors postulate plasma generation at a fixed point in space (see also Fig. 3). Indeed, the occurrence of plasma formation at a fixed point in space implies focussing of the laser beam. However, in principle a femtosecond laser beams which propagates over long distances 
according to the mechanism described in section 2 has no true focus. A 2-D representation of the radially symmetric plasma emission, as assumed in the present report, is indicated by red arrows in Fig. 3.

In order to estimate the power of the plasma emission, we make use of the data from [5], in which the light intensity of a high-power femtosecond laser beam was determined as a function of distance (see Fig. 4). The energy lost by the laser beam per travelled unit of distance constitutes an upper bound for the energy dissipated in the form of plasma radiation. We assume, on the basis of the dashed line in Fig. 4, an intensity loss of 5\% per $100 \mathrm{~m}$ of travelling distance. If we take an average laser power of $10 \mathrm{~W}$ (as in section 3.1), then a power of $5 \mathrm{~mW}$ is dissipated per meter.

In the countermeasure scenario, the ground-based laser is directed in such a way that it passes directly in front of the seekerhead of the missile, in order that $50 \%$ of the emitted plasma radiation enters the seekerhead. The other $50 \%$ radiates away from the missile. If we now assume a seekerhead diameter of $10 \mathrm{~cm}$, we see that the equivalent irradiance of the seekerhead is about $3 \cdot 10^{-2} \mathrm{~W} / \mathrm{m}^{2}$. (The seekerhead diameter is relevant here for the comparison of irradiances because of the geometry: we want to calculate the equivalent irradiance on the detector area of a line-shaped laser beam.) However, only part of this energy is in the relevant spectral region of 3-5 $\mu \mathrm{m}$. For an assumed plasma temperature of $10000 \mathrm{~K}$ (basing on [13]) this percentage is $0.34 \%$, which would give a seekerhead irradiance of $9 \cdot 10^{-5} \mathrm{~W} / \mathrm{m}^{2}$. The value is in the same order of magnitude as the signature of a plane at $1000 \mathrm{~m}$, which is $10^{-3}-10^{-5} \mathrm{~W} / \mathrm{m}^{2}$ (see section 3.1). Given (i) the very optimistic parameter values used in the estimation, (ii) the little realistic assumption of fully efficient plasma generation, and (iii) the fact that the seekerhead irradiance from the countermeasure device should exceed the plane signature by a few orders of magnitude, we can conclude that plasma generation by high-power femtosecond lasers is not promising for countermeasure applications. Especially the use of femtosecond lasers to create flare-like objects, as proposed in [12], is not realistic.

\subsection{Laser beam filamentation and microwave guidance}

Ionisation of the air in high-power femtosecond laser beams occurs at intensities of around $4 \cdot 10^{13} \mathrm{~W} / \mathrm{cm}^{2}$ [4]. Because of the defocussing of the light by the generated plasma, higher light intensities are not possible in air. However, one can easily increase the laser power. From this, it follows that on exceeding the plasma threshold, the laser beam cross section should somehow increase. As mentioned in Section 2, this is accomplished by generating a number of filaments which is approximately equal to $I / I_{\text {critical }}$, in which I signifies the light intensity and $I_{\text {critical }}$ is the critical light intensity for plasma generation.

A possible application of these plasma filaments is related to the guidance of microwaves. Microwaves are potentially important for countermeasure applications. Raytheon proposed a system using High Power Microwaves (HPM) as a countermeasure against infrared seekers [14]. In their 'Vigilant Eagle' concept, high power radio frequency (RF) beams from ground-based transmitters interact with the seekers of infrared guided missiles. Using a waveguide to transport the RF power over a large range with less losses would be an interesting option. This waveguide should be highly manoeuvrable in order to track a threat missile. A laser based waveguide could fulfil this requirement. An additional advantage of such a waveguide would be that it reduces the potential risk of hitting the aircraft with side lobes of the HPM beam.

Musin et al. [15] propose the use of arrays of femtosecond-laser-induced filaments as a waveguide, based on the fact that the refractive index and the absorption coefficient in and between the filaments differ. The gain in power on the target by using a filament waveguide is a compromise between, on one hand, the waveguide gain compared to free space propagation and, on the other hand, the waveguide transmission losses. Musin et al. [15] estimate the propagation distances for which intensity enhancement can be realised to be of the order of hundreds of meters. In these estimations long-range radiation losses were neglected. The estimated range is an order of magnitude less than the kilometer-size propagation distances which are necessary for practical countermeasure applications. Furthermore, in order for the waveguide to function properly, it should be stable in time and consist of regularly spaced filaments. Unfortunately, the beam profile of a femtosecond laser is not fixed and the filament position distribution has a random character [16]. We therefore see that with the present level of technology it will not be possible to successfully employ plasma filaments, generated by femtosecond laser beams, for waveguiding purposes in countermeasure applications. In order to fully take advantage of theoretical possibilities, considerable advances in the field of filament control should be made. 


\subsection{Femtosecond laser damaging of detectors}

Theoretical models of laser-induced damage (LID) of solids distinguish between extrinsic and intrinsic damaging mechanisms [17]. Extrinsic damage is related to heating of a material which is affected by defects such as impurities and inclusions. Intrinsic damaging of higher-quality material occurs mainly via two processes: impact ionisation and multiphoton ionisation. It was argued in Ref. [18] that ultra-short pulses (below $10 \mathrm{ps)} \mathrm{lead} \mathrm{to} \mathrm{ablation} \mathrm{of} \mathrm{material} \mathrm{via} \mathrm{intrinsic}$ damaging mechanisms, whereas longer pulses (above $100 \mathrm{ps}$ ) tend to lead to conventional melting and/or crackformation via extrinsic damaging mechanisms. However, no clear consensus concerning exact mechanisms exists in the literature, see also [17]

In the context of countermeasure applications it is not clear which damaging mechanisms are preferable. On one hand, larger-scale damage type such as cracking and melting could be desirable because of their obviously devastating impact on optical component properties. On the other hand, more subtle treatment of optical component with femtosecond laser beams, peeling off layers via ablation mechanisms, might be faster or energetically favourable. Illustrations of these different types of damage mechanisms can be found in [19], discussing homogeneous, ablation-like damage, and [20], where more coarse and irregular damage is reported.

In spite of these unresolved uncertainties concerning mechanisms, experimental trends are less controversial. The LID threshold, i.e. the laser pulse energy at which damage of optical material occurs, becomes lower for shorter pulses. This is illustrated in Fig. 9 of [17], in which experimental observations of the damage threshold of $\mathrm{SiO}_{2}$ layers are compared to a model. The lower LID threshold for ultra-short pulses (such as femtosecond pulses) is related to the fact that more electrons are excited in a smaller volume. Indeed, since thermalisation processes have time constants in the picosecond range, thermal diffusion is significantly reduced for pulse lengths as short as femtoseconds [17]. The expansion of the non-equilibrium electron gas may give rise to high pressures and, consequently, considerable shock waves [21].

For countermeasure applications both types of damage mentioned in the previous paragraph are potentially useful, since they lead to obstruction of the transmission of optical components. Much depends on the laser parameters, such as geometrical size of the laser spot and optical power. Also important is the laser wavelength, which preferably should be efficiently absorbed by the material of which the optical component is made. This means that a germanium filter should ideally be irradiated with a wavelength outside the transmission window of 2-20 $\mu \mathrm{m}$. However, if irradiation intensities are sufficiently high, then multi-photon absorption processes may lead to enhanced absorption at wavelengths which are non-resonant with the absorption spectrum of the optical component. This means that high-power femtosecond laser pulses, which are at present mostly centered around a wavelength of $800 \mathrm{~nm}$, can also be effective for countermeasures in the UV spectral range.

Based on available reports, we conclude that femtosecond laser pulses are promising for countermeasure applications, but still a substantial amount of research is needed to arrive at a point where applications become realistic. A potential advantage of femtosecond pulses with respect to longer pulses is long-distance propagation of filaments, which may give rise to a larger effective range of the countermeasure.

\subsection{Femtosecond laser jamming of detectors}

Damaging of detector materials, as discussed in the previous section, requires high optical powers. It may be difficult to achieve sufficiently high powers for physical damage in countermeasure scenarios, in which attenuation processes (via scattering and absorption of the atmosphere) can prevent obtaining the desired result. However, interaction between femtosecond laser pulses and photonic detectors can also be useful for countermeasure applications at lower powers than those required for damaging, via so-called bleaching of the detector material [22,23].

In this process, the photon burst of the femtosecond laser leads to a large concentration of excited charge carriers, which in turn saturates the absorption of new photons. This is possible because carrier recombination occurs at a much slower rate than the femtosecond excitation: carriers are generated much faster than they can recombine. As a result the carrier concentration becomes very high, and the lowest levels of the density of states of the semiconductor detector material (near the band gap) can no longer accommodate all excited charge carriers. As a consequence energetically higher states start to be filled. This process continues until the photon energy of the exciting light is reached. After this point, no free states are available for excited carriers. Absorption is no longer possible and the detector ceases to report incoming 
photons. Femtosecond pulses are essential for this process, since recombination processes in semiconductor materials have time constants which are typically in the picosecond-to-nanosecond range. This means that femtosecond laser pulses are sufficiently short to lead to a carrier concentration surge.

The described mechanism is especially useful against focal-plane-based imaging seekers, which are relatively insensitive to temporally modulated signals. The femtosecond jamming approach prevents target photons from being observed by the individual pixels of the array. However, the jamming mechanism associated with a single pulse only works temporally, since the detector returns to its initial state in a time comparable to the recombination time. Given a carrier recombination time of $10 \mathrm{~ns}$, this means that the femtosecond laser in principle has to operate at a multi-MHz repetition rate in order to jam the detector continuously. Faster carrier recombination times, which are certainly feasible, lead to even higher required laser repetition rates. At present, femtosecond laser technology is compatible with operation in the $\mathrm{kHz}$ range. This poses a potential challenge for effective femtosecond laser jamming. However, since further mechanisms may also be involved in the interaction between femotsecond laser pulses and semiconductor detectors, only thorough experimental investigations can reveal the potential of femtosecond jamming. Other important factors relevant to the effectiveness of the jamming mechanism are the band gap of the detector material and the laser wavelength. Experimental investigations of the femtosecond jamming mechanism are still in the preliminary phase.

\section{DISCUSSION AND CONCLUSIONS}

In the present report we investigated the potential applications of high-pulse-power femtosecond laser beams in a variety of countermeasure applications. We paid attention to the following aspects of femtosecond laser beams: (i) the whitelight spectral continuum, (ii) plasma emission, (iii) beam filamentation, (iv) high-power damaging of components, and (v) jamming of detectors.

We saw that application of femtosecond laser beams for dazzling of missile seeker heads is not at all promising, neither via white-light generation in the ultra-short laser pulses nor via glowing plasma. In both these cases the generated light intensity is too low to successfully prevail over the signal of a typical target. The white light which is generated in the laser because of a process which is labelled 'self-phase modulation' has the additional disadvantage that it is highly directional, which implies that for successful countermeasures head-on engagement of the laser beam with the seeker head is required. Indeed, in that case one could also use a MWIR laser to dazzle the seeker. This means that groundbased countermeasure applications based on white-light generation by femtosecond laser systems would be highly ineffective. Glowing plasma, created along the whole laser beam path and radiating to all sides, would not be hindered by angular restrictions. However, as was shown in this report, the most optimistic estimates of the radiated power of such plasma filaments are not high enough to compete with a typical target.

Filamentation of the femtosecond laser beam, which is in essence due to self-focussing effects, has a theoretical application in the waveguiding of radiofrequency (RF) beams which function as countermeasures. Indeed, because of the variation of the index of refraction between areas with and without filaments, suitably positioned filaments can constitute a RF waveguide. However, it turns out that the propagation distances for which intensity enhancement can be realised are of the order of a few hundred meters at best, which is not sufficient for most practical countermeasure applications. Also, at the present level of technology the radial filament distribution is not stable in time and is difficult to control, which for the time being makes implementation of femtosecond laser beams as RF waveguides unfeasible.

The most promising application of femtosecond laser beams in the field of countermeasure applications is related to its high-power features. As the pulse length of a laser pulse decreases, the damage threshold energy of optical components also decreases. Therefore, short laser pulses such as femtosecond pulses can in principle provide an energetically favourable manner to induce damage. Furthermore, short laser pulses (under $\sim 100 \mathrm{ps}$ ) in general give rise to a different type of damage morphology than longer laser pulses (over $\sim 100 \mathrm{ps}$ ): shorter pulses lead to ablation processes, whereas longer pulses lead to cracking. It is an open question which damaging technique should be preferred for countermeasure applications. At this moment the exact damage mechanisms in different materials and their consequences for countermeasures are not yet fully understood and are the subject of ongoing research. The same is true for femtosecond jamming, which relies on bleaching of the detector material: high carrier concentrations are generated in the detector material in order to obstruct further photon absorption. Experimental investigation of these processes is in progress.

Furthermore, an important issue is the practicality of the required systems, which should not be too large or heavy to handle. For example, a container-sized femtosecond laser system is too bulky to be used for defense purposes in dynamic 
scenarios. Nevertheless, taking into account these challenges, femtosecond lasers can be expected to show their additional value for countermeasure applications in the near future.

\section{REFERENCES}

[1] Teramobile website: www.teramobile.org

[2] M. Châteauneuf and J. Dubois, 'Canadian defense agency acquires new portable terawatt laser', SPIE Newsroom 10.1117/2.1200612.0506 (2006).

[3] G.A. Mourou, C.P.J. Barty, and M.D. Perry, 'Ultra-high intensity lasers: physics of the extreme on a tabletop', Physics Today 51, 22 (1998).

[4] J. Kasparian, R. Sauerbrey, and S.L. Chin, 'The critical laser intensity of self-guided light filaments in air', Appl. Phys. B 71, 877 (2000).

[5] G. Méchain, C. D’Amico, Y.-B. André, S. Tzortzakis, M. Franco, B. Prade, A. Mysyrowicz, A. Couairon, E. Salmon, and R. Sauerbrey, 'Range of plasma filaments created in air by a multi-terawatt femtosecond laser', Opt. Comm. 247, 171 (2005).

[6] J. Kasparian, 'Filaments of light', Am. Sci. 94, 150 (2006).

[7] W. Demtröder, Laser Spectroscopy, p. 614, Springer Berlin (1996).

[8] J. Kasparian, R. Sauerbrey, D. Mondelain, S. Niedermeier, J. Yu, J.-P. Wolf, Y.-B. André, M. Franco, B. Prade, S. Tzortzakis, A. Mysyrowicz, M. Rodriguez, H. Wille, and L. Wöste, 'Infrared extension of the supercontinuum generated by femtosecond terawatt laser pulses propagating in the atmosphere', Opt. Lett. 25, 1397 (2000).

[9] J. Yu, D. Mondelain, G. Ange, R. Volk, S. Niedermaier, J. P. Wolf, J. Kasparian, and R. Sauerbrey, 'Backward supercontinuum emission from a filament generated by ultrashort laser pulses in air', Opt. Lett. 26, 533 (2001).

[10] M. Rodriguez Langlotz, 'Terawatt-Femtosekunden-Laserpulse in der Atmosphäre: Phänomene und Anwendungen', Ph.D. thesis, Free University Berlin (2004).

[11] R.A. Lamb, K. Cook, and A.K. Kar, 'Phase stability and diffraction effects in self-focused white light filaments in water and glass', Proc. SPIE 5620, 218 (2004).

[12] W. Bohn and H.-A. Eckel, 'Device and method for protection against missiles and use of a laser device', Patent EP1746381 (2007).

[13] J.H. Jebsen and G. Aknine, 'Plasma flare IR and UV emitting devices', Patent WO2005026650 (2005).

[14] Raytheon product brochure RMS 4/06 4K 60259R: 'Vigilant Eagle Airport Protection System; Ground-based Counter-MANPADS Protection for Large Airports and Air Bases'. Available via www.raytheon.com.

[15] R. R. Musin, M.N. Shneider, A.M. Zheltikov, R.B. Miles, 'Guiding radar signals by arrays of laser-induced filaments: finite-difference analysis', Appl. Opt. 46, 5593 (2007).

[16] L. Bergé, S. Skupin, F. Lederer, G. Méjean, J. Yu, J. Kasparian, E. Salmon, J.P. Wolf, M. Rodriguez, L. Wöste, R. Bourayou, and R. Sauerbrey, Phys. Rev. Lett. 92, 225002 (2004).

[17] A.A. Manenkov, Proc. SPIE 7132, 713202 (2008).

[18] B.C. Stuart, M.D. Feit, A.M. Rubenchik, B.W. Shore, and M.D. Perry, 'Laser-Induced Damage in Dielectrics with Nanosecond to Subpicosecond Pulses', Phys. Rev. Lett. 74, 2248 (1995).

[19] P. Bourdon, H. Bürsing, B. Eberle, R. Ebert, T. Marrel, A. Mysyrowicz, and O. Vasseur, 'Interaction of high peak power femtosecond laser pulses with optronic equipments', OPTRO2005.

[20] P.-Y. Madec, E. Idiart-Barsoum, O. Meyer, P. Chardard, and C. Leonet, 'Presentation of COMETE: A Medium Energy Counter-Measure Testbed', OPTRO2005.

[21] R. Bernath, C.G. Brown, J. Aspiotis, M. Fisher, and M. Richardson, 'Shock-wave generation in transparent media from ultra-fast lasers', Proc. SPIE 6219, 62190A (2006).

[22] M.K. Rafailov, 'Ultra-fast Bandgap Photonics: Sensing and Counter-Sensing Capabilities', Proc. SPIE 7055, 70550J (2008).

[23] M.K. Rafailov, ‘Ultra-fast Laser IR Countermeasures’, Proc. SPIE 7325, 73250W (2009). 